\title{
Establishment of a novel therapeutic vector targeting the trigeminal ganglion in rats
}

This article was published in the following Dove Press journal:

Drug Design, Development and Therapy

4 February 2016

Number of times this article has been viewed

\author{
Kun $X u^{1,2}$ \\ Shi-Yin Pan ${ }^{1,2}$ \\ Jin-Xin Song' \\ Xian-Ning Liu ${ }^{1,2}$ \\ $\mathrm{Na} \mathrm{An}^{1,2}$ \\ Xuan Zheng ${ }^{1,2}$ \\ 'Department of Ophthalmology, \\ The No I Hospital of Xi'an, ${ }^{2}$ Shaanxi \\ Provincial Key Laboratory of \\ Ophthalmology, Ophthalmological \\ Institute of Shaanxi Province, Xi'an, \\ Shaanxi Province, People's Republic \\ of China
}

Background: In the pathogenesis of herpes simplex keratitis, herpes simplex virus type 1 (HSV-1) infection begins in corneal epithelium cells and then progresses through the sensory nerve endings and finally travels up forward to the trigeminal ganglion (TG), where it remains as latent virus. The available anti-HSV therapies do not completely suppress the recurrence of active HSV-1 infection. The aim of this study was to establish a novel replication-defective (rd) HSV-1 (rdHSV) vector (rdHSV-interferon gamma [IFN $\gamma]$ ) that could effectively target the TG.

Methods: Recombinant HSV-1 virus was inserted into a shuttle plasmid carrying IFN $\gamma$ to establish the rdHSV-IFN $\gamma$ vector. Safety was evaluated in vitro by $50 \%$ cellular cytotoxicity in transfected SH-SY5Y neuroblastoma cells and in vivo by Kaplan-Meier survival estimate and infection rate. Wistar rats were immunized with rdHSV-IFN $\gamma$ to evaluate the TG targeting efficiency. Real-time polymerase chain reaction and Western blot assays were used to evaluate IFN $\gamma$ mRNA and protein expression and rdHSV-IFN $\gamma$ localization.

Results: The rdHSV-IFN $\gamma$ vector was successfully constructed and showed high in vitro safety and overall survival and a corneal infection rate similar to that of control rats immunized with saline (control group; $P>0.05$ ). Real-time polymerase chain reaction and immunohistochemistry assays confirmed IFN $\gamma$ expression and effective TG targeting on days 14 and 21, which increased with postimmunization time. Moreover, IFN $\gamma$ was expressed sufficiently in the TG tissues.

Conclusion: The rdHSV-IFN $\gamma$ can act as an effective gene transporting vector that carries the therapeutic genes to the TG and triggers its expression.

Keywords: replication-defective HSV-1, interferon gamma, trigeminal ganglion, therapeutic gene

\section{Introduction}

Herpes simplex virus (HSV) infection in the corneas is the main kind of keratitis, which always develops to a chronic or an acute corneal inflammation. ${ }^{1,2}$ Among several HSVs, the herpes simplex virus type 1 (HSV-1) is the most common cause of the herpes simplex keratitis (HSK). The HSK is a prevalent reason for corneal blindness in the modern clinical ophthalmology. ${ }^{3}$ Regarding the pathology of the HSK, the HSV-1 first infects the corneal epithelium cells, then invades the sensory nerve endings, and finally travels to the trigeminal ganglion (TG). ${ }^{4}$ When the HSV-1 passes the acute stage, it becomes latent in the TG. ${ }^{4}$ It is well known that HSK is one of the most frequently recurrent corneal diseases based on the reactivation of the latent HSV-1 in the trigeminal sensory neurons. The recurrence rate of HSK within 2 years is $\sim 23 \%-33 \%$. Approximately $20 \%-25 \%$ of the recurrent HSK could develop to the T-cell-mediated HSK, which is the prevalent reason for the unilateral blindness. ${ }^{5}$

Although some anti-HSV or immunoregulatory treatments are available, they do not eliminate the latent virus and therefore cannot completely suppress the recurrence of the HSV-1 infection. ${ }^{6}$ Recently, DNA vaccines or vectors have been shown to trigger a cell-mediated immune response in the HSK rat models. However, HSV-1 infection
Correspondence: Kun Xu; Jin-Xin Song Department of Ophthalmology, The No I Hospital of Xi'an, Fen Rd 30, The South Street, Xi'an, Shaanxi Province, People's Republic of China

Tel +862987630869

Fax +862987241606

Email xukun04I8@I26.com;

jinxxsong@yeah.net
Drug Design, Development and Therapy 2016:10 585-592

585

Dovepress

http://dx.doi.org/10.2147/DDDT.S96730 (c) (i) (5) 2016 Xu et al. This work is published and licensed by Dove Medical Press Limited. The full terms of this license are available at https://www.dovepress.com/terms.php hereby accept the Terms. Non-commercial uses of the work are permitted without any furcher permission from Dove Medical Press limited, provided the work is properly attributed. For permission for commerial ef of this work percial uses of the work are permitted without any further permission from Dove 
was blocked only in the acute stage of HSK and there was no effect against the latent HSV-1 infection of the TG. Hu et $\mathrm{al}^{7}$ developed a DNA vaccine that triggered a better immune protection against the HSV-1 infection of the ocular surface but did not inhibit the recurrence of corneal HSV-1 infection. Over the past 20 years, investigations to develop therapies for HSV-1 infection have been focused on developing effective treatment on the removal of latent infection of the TG and on the elimination of the latent virus in the TG. In this study, we attempted to establish an effective therapeutic system targeting the TG and cleaning up the latent virus in the TG. We took advantage of the neurotrophic properties of HSV-1 to make a recombinant, replication-defective (rd) HSV-1 vector that could be efficiently transported along neurons and could carry therapeutic IFN to the TG without any toxicity.

\section{Materials and methods}

\section{Animals, cell lines, and viruses}

Male specific pathogen-free Wistar rats aged 5-6 weeks were purchased from the Experimental Animal Center of Xi'an Jiaotong University (Xi'an, People's Republic of China). The rats were raised under pathogen-free conditions in containers supplied with filtered air. The Ethics Committee of the No 1 Hospital of Xi'an, Xi'an, People's Republic of China, approved all the animal experiments.

The human neuroblastoma cell line SH-SY5Y and Vero cells were obtained from the Chinese Center for Disease Control and Prevention (Beijing, People's Republic of China), grown in a $5 \% \mathrm{CO}_{2}$ atmosphere at $37^{\circ} \mathrm{C}$ in Dulbecco's Modified Eagle's Medium (DMEM) supplemented with 10\% fetal bovine serum, $100 \mu \mathrm{g} / \mathrm{mL}$ streptomycin sulfate, and 100 units/mL penicillin G sodium.

The HSV-1 strain SM44 was obtained from Professor Xiuping Zhu at the Ophthalmological Institute of Shaanxi Province (Xi'an, People's Republic of China) and was propagated, activated, and cultured in Vero cells. No ethics statement was required from the institutional review board for the use of these cell lines.

\section{Recombination of rdHSV-I}

To control the replication of the established vector, we removed the immediate-early, ICP27, ICP4, and ICP34.5 genes, which are essential for replication, to form the rd viruses. ${ }^{8}$ In order to inhibit the cytotoxicity of the rdHSV-1, the pathogenic gene, VP16, was inactivated and mutated. ${ }^{9}$ To allow prolonged expression of the exogenous genes, we inserted a strong heterologous promoter at the location of the $1.4 \mathrm{~kb}$ downstream under the latency-associated promoter 1 TATA box (Figure 1A).

\section{IFN $\gamma$ clone and establishment of rdHSV-I therapeutic system}

Lymphocytes were isolated from the blood of a healthy volunteer and were grown in primary culture in the DMEM solution supplemented with $10 \%$ fetal bovine serum. The HSV-1 strain SM44 virus was used to activate the lymphocytes, and the total RNA was extracted from the activated lymphocytes. The total RNA was employed to synthesize the cDNA. IFN $\gamma$ was amplified from the cDNA by PCR using 5'-ACGAAGCTTATGAAATATACAAGTTATATCTTG$3^{\prime}$ as the forward primer and $5^{\prime}$-ATCCTCGAGTTACTGGG ATGCTCTTCGAC- ${ }^{\prime}$ as the reverse primer.

The amplified $I F N \gamma$ gene was cloned and inserted into a shuttle plasmid (Figure 1B). The rdHSV-1 vector was generated by calcium phosphate cotransfection of complementing cells with the shuttle plasmid and HSV-1 backbone as previously described. ${ }^{9,10}$ The rdHSV-1 system (rdHSVIFN $\gamma$ ) was established successfully (Figure 1C), and titers were evaluated by using tenfold serial dilution as previously reported. ${ }^{11}$ The plaque forming units (PFUs) typically ranged from $3 \times 10^{8} \mathrm{PFU} / \mathrm{mL}$ to $2 \times 10^{9} \mathrm{PFU} / \mathrm{mL}$.

\section{XTT cytotoxicity assay}

SH-SY5Y cells cultured in 96- or six-well plates were infected with the rdHSV-IFN $\gamma$ vector (at final concentrations of $25 \mathrm{TCID}_{50}, 50 \mathrm{TCID}_{50}, 100 \mathrm{TCID}_{50}$, and $200 \mathrm{TCID}_{50}$, respectively). After 48 hours, antiviral activity and cytotoxicity were determined by XTT assay. The dimethyl sulfoxide solubilization solution was used as a negative control, and the assay was performed as previously described. ${ }^{12}$ The results of XTT assay were reported as the percentage inhibition of cytotoxicity calculated as follows: inhibition $\%=[100-(A /$ $\left.\left.A_{s}\right) \times 100\right] \%$, where $A_{t} / A_{s}$ is the ratio of the absorbance of the formazan-containing assay solution to the absorbance of the XTT solubilization solution at 48 hours. The vector concentration resulting in $50 \%$ cellular cytotoxicity $\left(\mathrm{CC}_{50}\right)$ was determined as described by Chiang et al. ${ }^{13}$

\section{Immunization with rdHSV-IFN $\gamma$}

Wistar rats were anesthetized, and their corneas were scarified with a syringe needle ( $1 \mathrm{~mL}$ type) for at least ten times. The rats were then randomly assigned to three groups $(\mathrm{n}=20$ per group), including blank rdHSV vector group (blank rdHSV-1), rdHSV-IFN $\gamma$ group, and control (CN) group (treated with saline), that is, to the topical treatment of the cornea with $10 \mu \mathrm{L}$ of blank rdHSV $\left(2 \times 10^{6} \mathrm{PFU}\right)$, rdHSV$\operatorname{IFN} \gamma\left(2 \times 10^{6} \mathrm{PFU} / \mathrm{mL}\right)$, and a $\mathrm{CN}$ group given normal saline solution. 


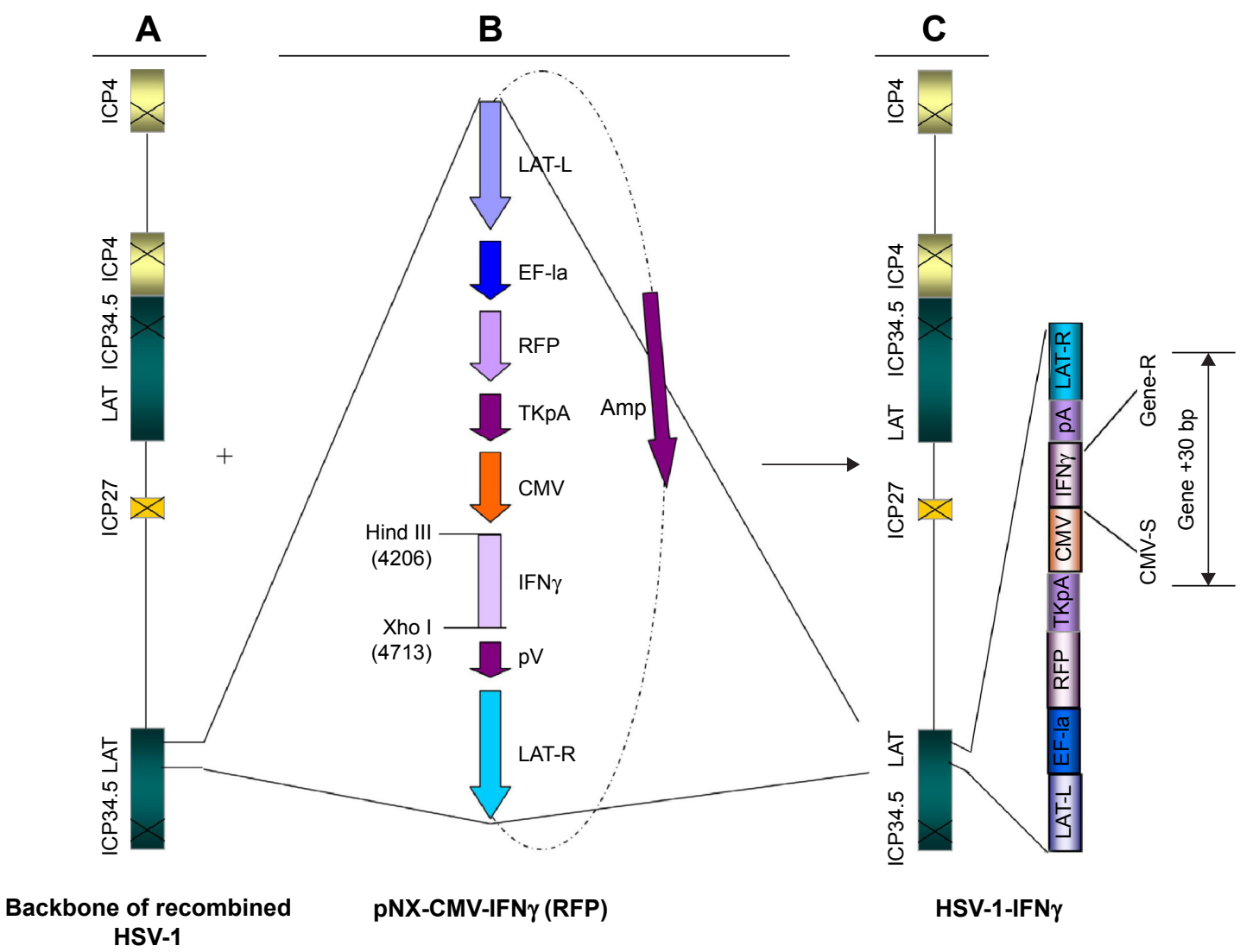

Figure I Construction of the rdHSV-IFN $\gamma$ therapeutic system.

Notes: (A) HSV backbone of the recombined HSV-I. (B) Shuttle plasmids carrying the IFN $\gamma$ gene. (C) The complete rdHSV-I-IFN $\gamma$ therapeutic system.

Abbreviations: HSV-I, herpes simplex virus type I; IFN $\gamma$, interferon gamma; rdHSV, replication-defective HSV-I; EF-I $\alpha$, elongation factor I alpha; CMV, cytomegalovirus promoter.

\section{Immunohistochemistry analysis}

Trigeminal ganglia were isolated from the rats, fixed in $4 \%$ paraformaldehyde, embedded in commercial tissue freezing medium (TIANGEN Biotech Co., Ltd., Beijing, People's Republic of China), and immediately frozen in isopentane. Cryosections $(4 \mu \mathrm{m})$ were cut, fixed in $70 \%$ ethanol, and incubated with mouse anti-HSV-1 monoclonal antibody (1:1,000; sc-57862; Santa Cruz Biotechnology Inc., Dallas, TX, USA) for at least 1 hour. The sections were also stained with hematoxylin and eosin, and all the procedures were performed according to the manufacturer's instructions.

\section{IFN $\gamma$ detection by Western blot assay}

Trigeminal ganglia were isolated for the detection of IFN $\gamma$ expression by Western blot assay. The treatment of trigeminal ganglia and the Western blot processes were performed according to the previous study. ${ }^{14}$ The primary antibodies were mouse anti-IFN $\gamma$ monoclonal antibody $(1: 2,000)$ and mouse anti- $\beta$-actin monoclonal antibody $(1: 2,000)$. The secondary antibody was horseradish-peroxidase-conjugated rabbit anti-mouse antibody (1:1,000; all from Santa Cruz
Biotechnology Inc.). The Western blot bands were scanned, and the pixel count and intensity of each band were semiquantified. The signals were normalized against $\beta$-actin, and the data were expressed as the percentage of the negative control signals.

\section{IFN $\gamma$ assay by real-time polymerase chain reaction}

The expression of IFN $\gamma$ mRNA in trigeminal ganglia was assayed by real-time polymerase chain reaction (RT-PCR). The forward primer was $5^{\prime}$-ACGAAGCTTATGAAATA TACAAGTTATATCTTG-3' ${ }^{\prime}$, and the reverse primer was 5'-ATCCTCGAGTTACTGGGATGCTCTTCGAC-3'. $\beta$-actin was used as the internal control; the forward primer was $5^{\prime}$-GGACTTC GAGCAGGAGATGG-3', and the reverse primer was 5'-GCACCGTG TTGGCGTAGAGG-3' The isolation of total RNA and cDNA syntheses was carried out using a commercial kit (TIANGEN Biotech Co., Ltd.). The procedure and conditions of the RT-PCR were followed according to the requirement or the instruction of the PCR reaction kit (Takara Biotechnology Co., Ltd., Dalian, 
People's Republic of China), using the ABI7500 RT-PCR System (Thermo Fisher Scientific, Waltham, MA, USA). The amplified DNA was run on $1.5 \%$ agarose gels; images were then digitally captured with a charge-coupled device camera and analyzed with an imaging analysis system.

\section{Statistical analysis}

The analyses were performed using SPSS 20.0 statistical software (IBM Corporation, Armonk, NY, USA). Student's $t$-test was used to evaluate the significance of differences between groups. $P$-values $<0.05$ were statistically significant.

\section{Results}

\section{Safety evaluation for rdHSV-IFN $\gamma$}

As therapeutic vectors must display high and proven safety, we evaluated the safety of rdHSV-IFN $\gamma$ both in vivo and in vitro. There was no evidence of HSV-1 or HSV-1 infection in any of the specific pathogen-free rats when the study began (data not shown). First, we also evaluated the safety of the rdHSV-IFN $\gamma$ in vivo. Rats were immunized by the topical administration of drops containing rdHSV-IFN $\gamma$ in their scarified corneas (Figure 2A). Second, we examined the survival rate and infection rate of rdHSV-IFN $\gamma$-immunized rats. Kaplan-Meier estimate confirmed that cumulative
A

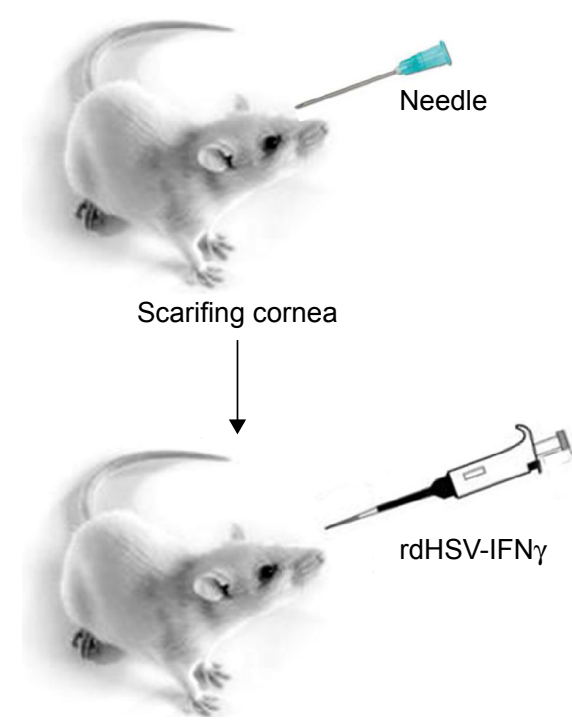

Immunization

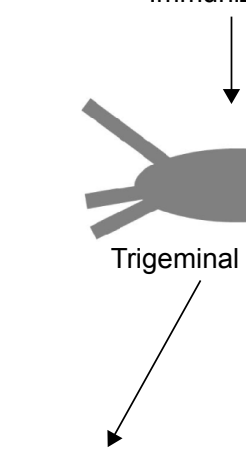

IFN $\gamma$ expression
Isolating
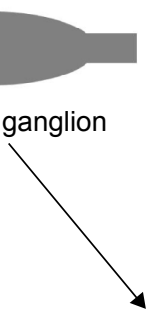

rdHSV targeting
B

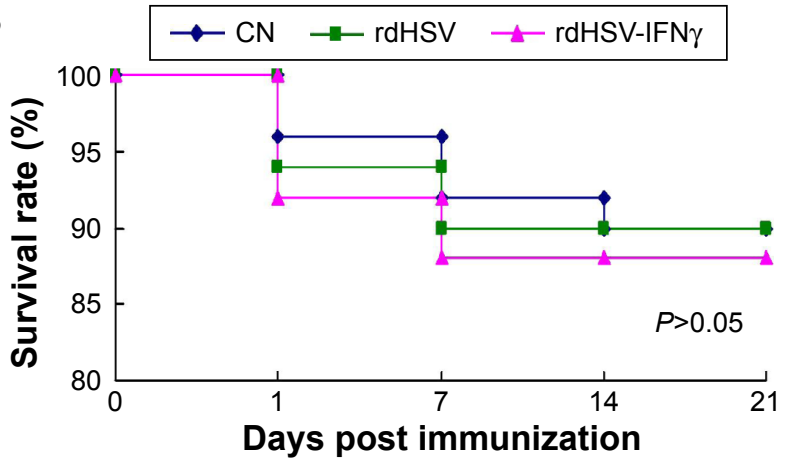

C

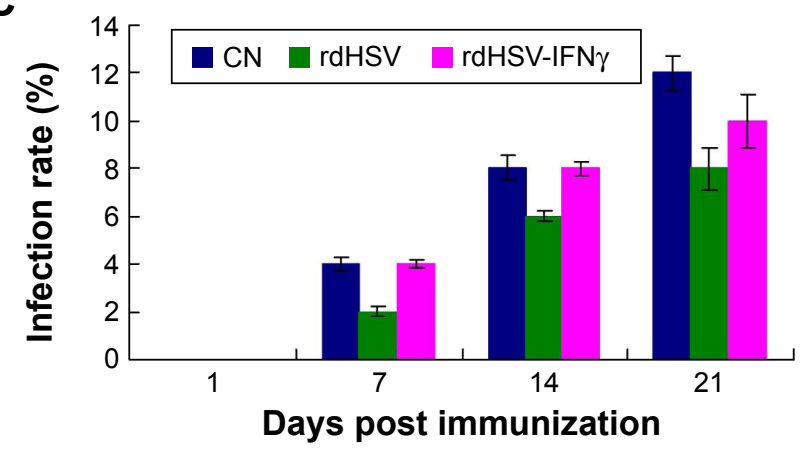

D

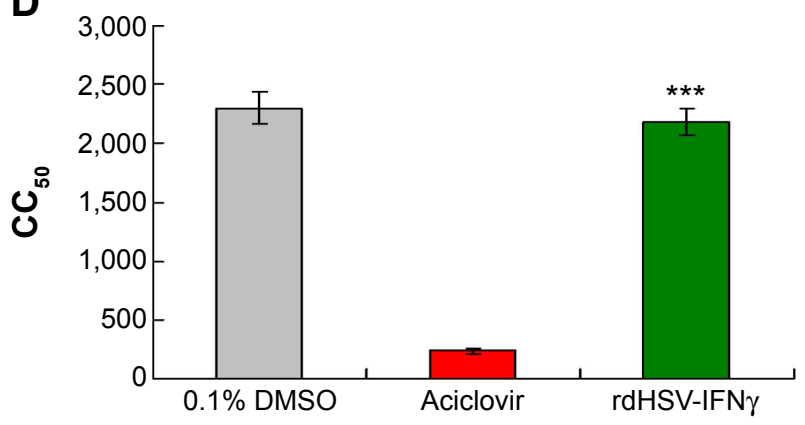

Figure 2 Safety evaluation of rdHSV-IFN $\gamma$ in vivo and in vitro.

Notes: (A) Immunization procedure for the rdHSV-IFN $\gamma$ vector. Corneas were scarified ten times with syringe needle (I mL type), and drops of rdHSV-IFN $\gamma$ solution were applied to the ocular mucosa. (B) Kaplan-Meier survival analysis for the three treatment groups. (C) Infection rates in the three treatment groups. (D) CC $\mathrm{C}_{50}$ o. I\% DMSO, aciclovir, and rdHSV-IFN $\gamma$. $* * * P<0.001, \mathrm{CC}_{50}$, or $\mathrm{EC}_{50}$ in the rdHSV-IFN $\gamma$ group vs the aciclovir group.

Abbreviations: $\mathrm{CC}_{50}, 50 \%$ cellular cytotoxicity; $\mathrm{CN}$, control; DMSO, dimethyl sulfoxide; $\mathrm{EC}_{50}$, $50 \%$ effective concentration; HSV-I, herpes simplex virus type I; IFN $\gamma$, interferon gamma; rdHSV, replication-defective HSV-I. 
survival in the rdHSV-IFN $\gamma$ group was equal to that in the $\mathrm{CN}$ group (Figure 2B, $P>0.05$ ). Furthermore, the side effects of rdHSV-IFN $\gamma$ treatment were also evaluated from day 1 to day 21 following the immunization and found no significant difference in the infection rate in the rdHSV-IFN $\gamma$ group compared with that in the $\mathrm{CN}$ group (Figure 2C).

In addition, the cytotoxicity of rdHSV-IFN $\gamma$ in SH-SY5Y cells, as indicated by the $\mathrm{CC}_{50}$ results, was significantly lower than that of aciclovir $(P<0.05)$ and not different from that of $0.1 \%$ dimethyl sulfoxide (Figure $2 \mathrm{D}, P>0.05$ ). Overall, the results support the safety of rdHSV-IFN $\gamma$ as a therapeutic system for the treatment of latent HSV-1 infection.

\section{Therapeutic rdHSV-IFN $\gamma$ effectively targets the TG}

The targeting efficiency of rdHSV-IFN $\gamma$ was assayed by the detection and amplification of the cloned IFN $\gamma$ protein. In this experiment, we isolated and detected the IFN $\gamma$ protein in cornea, trigeminal nerve, and TG on days 1, 7, 14, and 21 by RT-PCR. The IFN $\gamma$ mRNA was found in the cornea on days 1 and 7 , but the expression had significantly decreased by day 14 and day 21 compared with day 1 and day $7(P<0.05$, Figure 3). Meanwhile, IFN $\gamma \mathrm{mRNA}$ was not found in the TG on days 1 and 7 , but the expression increased significantly from day 7 to day 21 (Figure 3). On day 21, IFN $\gamma$ mRNAs were primarily distributed in the TG and were very low in the cornea. The pattern of IFN $\gamma$ mRNA distribution over time from day 1 to day 21 led us to conclude that rdHSV-IFN $\gamma$ could finally target the TG on day 21 , which may indicate that day 21 is optimal for the clearance of latent HSV-1 in the HSK rat models (Figure 3).

The rdHSV-IFN $\gamma$ targeting and expression were also characterized by immunohistochemistry (Figure 4). The results showed that IFN $\gamma$ protein expression in the cornea decreased significantly from day 1 to day 21 (Figure 4A). The IFN $\gamma$ protein was located mainly in the cornea on days 1 and 7 , but the level was significantly lower on days 14 and 21 . IFN $\gamma$ protein was expressed in the trigeminal nerve on days 7 and 14 but was no longer detected there on day 21 (Figure 4B). Most important, IFN $\gamma$ expression in the TG began on day 14 and rapidly reached a peak level on day 21 (Figure 4C). IFN $\gamma$ expression in the TG thus increased with time after immunization.

\section{Effective expression of rdHSV-IFN $\gamma$ in TG tissue}

The results described earlier indicated that the rdHSV-IFN $\gamma$ could effectively localize in TG tissue, where IFN $\gamma$ mRNA (Figure 5A) and protein expression (Figure 5B) were found to be the strongest on days 14 and 21 . The peak expression was coincident with the time that rdHSV-IFN $\gamma$ was present in the TG. The findings thus suggest that the rdHSV-IFN $\gamma$ not only targeted the TG tissues but also carried the therapeutic gene to the TG, where it could then clear the latent HSV-1.

\section{Discussion}

Key objectives of drug design are the effective targeting of the diseased region and the induction of protective responses that lead to significantly shortened duration of illness and relief of disease symptoms. ${ }^{7,15}$ In the present study, we constructed an rdHSV-1 vector with the potential to effectively target the latent HSV-1 in TG tissue. We also cloned a therapeutic antiviral IFN $\gamma$ gene fragment with the potential of increasing host immunogenicity.

Although there are many methods of delivering genes into mammalian cells in the past few decades, no specific vector targeting the trigeminal nerve or TG has been developed. ${ }^{16-18}$ Such a vector is especially important for
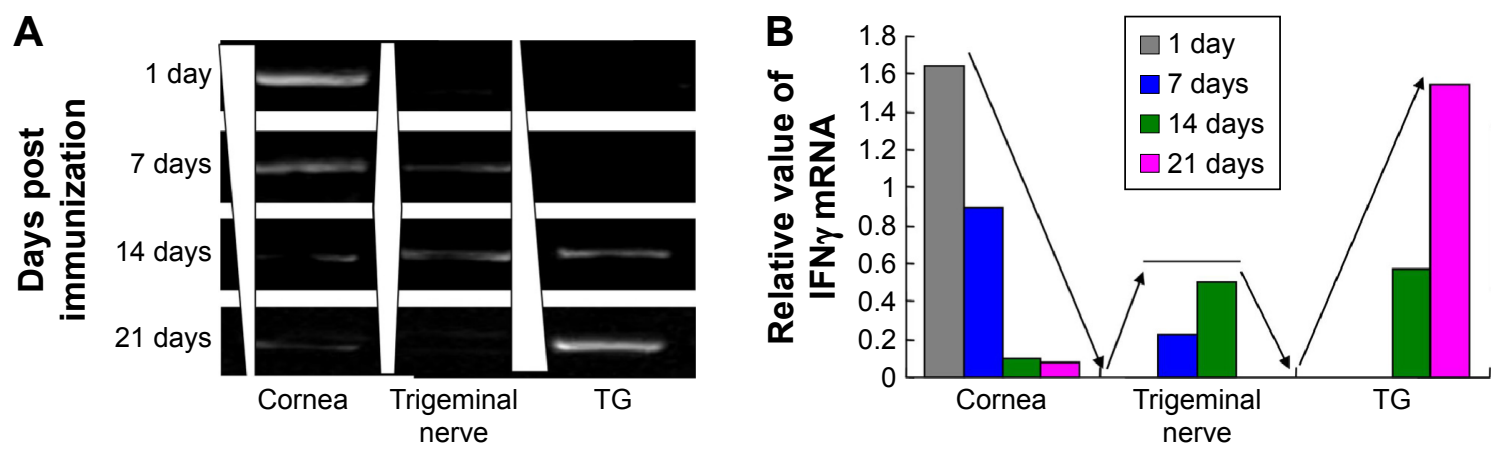

Figure 3 RT-PCR assay of mRNA expression in the cornea, trigeminal nerve, and TG from day I to day 21.

Notes: (A) IFN $\gamma$ mRNA on day I to day 21 post immunization in the cornea, trigeminal nerve, and TG. (B) Statistical analysis for change in IFN $\gamma$ mRNA expression in the cornea, trigeminal nerve, and TG from day I to day 21 .

Abbreviations: IFN $\gamma$, interferon gamma; RT-PCR, real-time polymerase chain reaction; TG, trigeminal ganglion. 


\section{rdHSV-IFN $\gamma$ targeting}

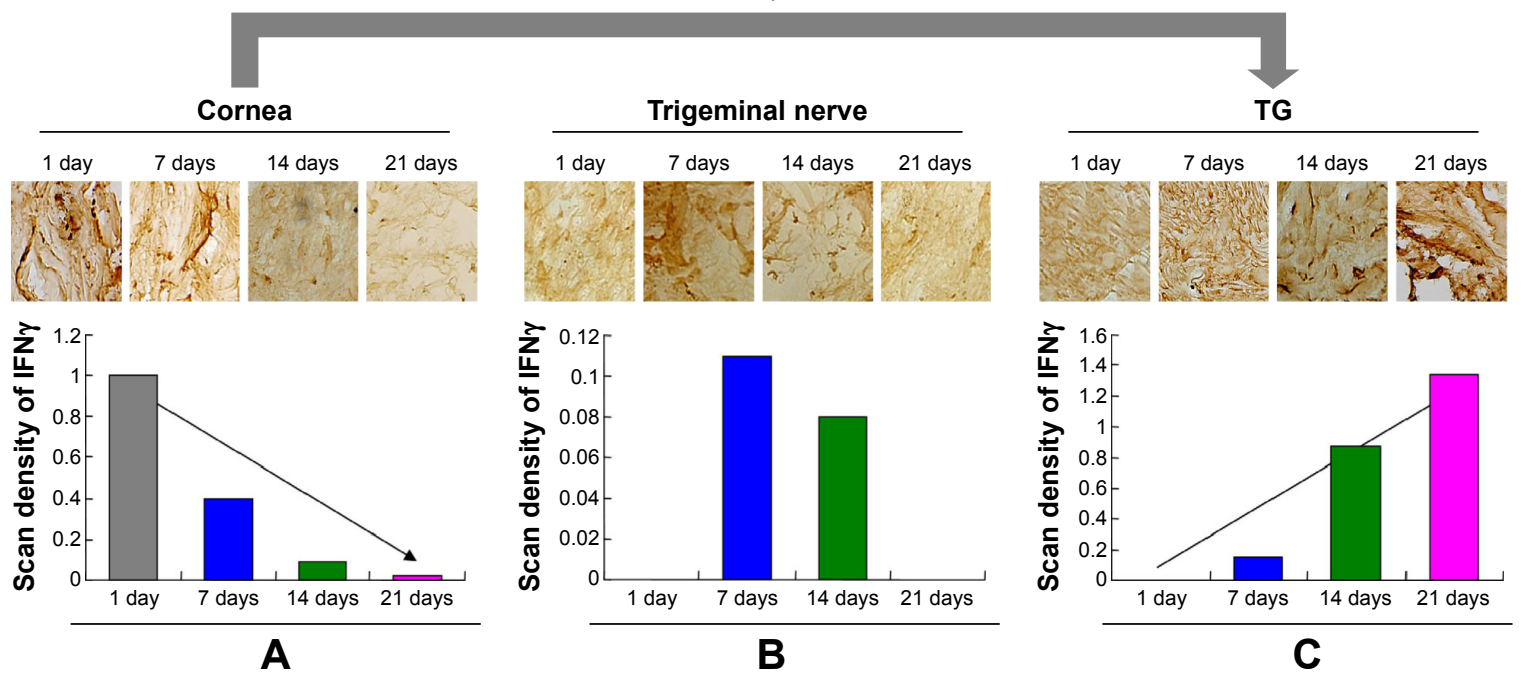

Figure 4 Immunohistochemical assay of IFN $\gamma$ protein localization in the cornea (A), trigeminal nerve (B), and TG (C) from day I to day 2 I post immunization.

Notes: The long arrow in the graph illustrates the direction of change in the expression of IFN $\gamma$ protein. The gray arrow illustrates the rdHSV-IFN $\gamma$ targeting pathway from cornea to TG.

Abbreviations: HSV-I, herpes simplex virus type I; IFN $\gamma$, interferon gamma; rdHSV, replication-defective HSV-I; TG, trigeminal ganglion.

treating HSK, because the available antiviral drugs do not target and eliminate the latent HSV-1 virus in the TG and thus cannot prevent recurrences. ${ }^{19,20}$ Therefore, we successfully constructed an rdHSV-1 vector system that could be used to effectively deliver therapeutic genes or immune regulators to the TG and clear the latent HSV-1.

In this study, we took an advantage of the neurotrophic properties of HSV-1 to reconstruct an rdHSV-1 vector that had lost its pathogenicity and ability to replicate and was effectively transported into the TG. For a clinically useful therapeutic tool, to confirm its safety in vitro, we transfected SH-SY5Y cells with the rdHSV vector. The results showed that rdHSV is very safe, with a $\mathrm{CC}_{50}$ six- to sevenfold higher than that of aciclovir, which is a drug having a wide clinical use. ${ }^{21}$ Actually, aciclovir has been used in ophthalmology practice for many years and is clinically effective with few side effects and low cytotoxicity for patients. ${ }^{22,23}$ Furthermore, we have also immunized the rdHSV-IFN $\gamma$ vector to the rats and examined the overall survival and the infection rate of the rats in every group. The results also indicated that
A

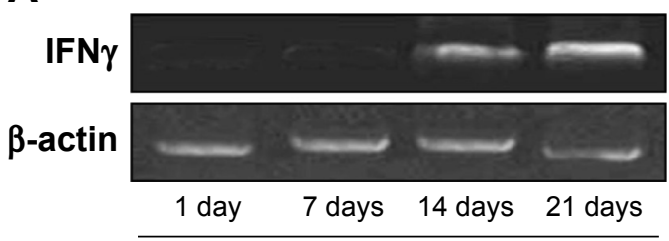

Days post immunization

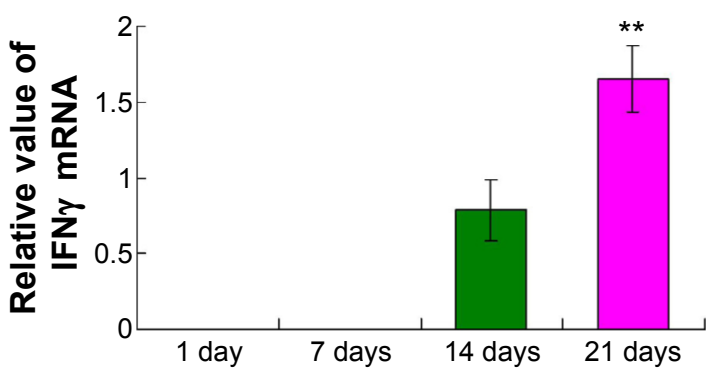

B

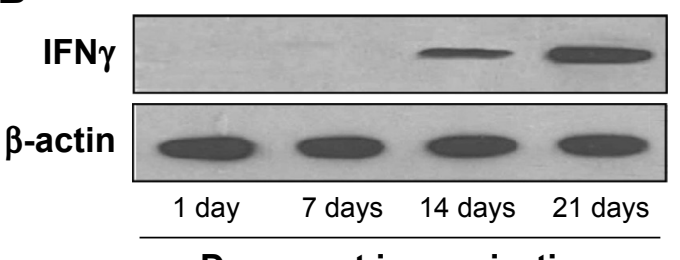

Days post immunization

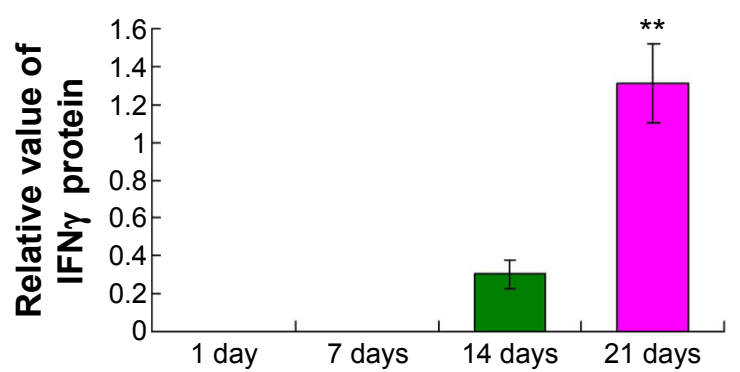

Figure 5 IFN $\gamma$ mRNA (A) and protein (B) expression in the TG tissues from day I to day 21 post immunization. Notes: ${ }^{* * P}<0.01$. IFN $\gamma$ mRNA or protein expression at 21 days vs 14 days.

Abbreviations: IFN $\gamma$, interferon gamma; mRNA, messenger RNA; TG, trigeminal ganglion. 
there was no significant difference between the rdHSV-IFN $\gamma$ vector treatment group and the $\mathrm{CN}$ group. The removal of pathogenic gene fragments and genes needed for replication prevented the occurrence of treatment-related side effects and wild-type viral infections previously seen with some recombinant HSV-1 vectors. ${ }^{24-27}$ The in vivo and in vitro experiments demonstrated the safety of rdHSV-IFN $\gamma$.

To evaluate the targeting effectiveness of rdHSV-IFN $\gamma$, we isolated trigeminal ganglia from immunized rats and assayed IFN $\gamma$ expression on days 1, 7, 14, and 21 after immunization. IFN $\gamma$ protein was expressed following immunization, and the expression level reflected the amount of rdHSV-IFN $\gamma$ contained in the TG. The IFN $\gamma$ mRNA and protein expression results indicate that rdHSV-IFN $\gamma$ targeted the TG, continuously following a pathway from the cornea to the trigeminal nerve and the TG. Therefore, the rdHSV-IFN $\gamma$ vector could effectively carry a therapeutic gene to HSV-1 virus-infected cells in the HSK rat model or HSK patients. This characteristic of the rdHSV-IFN $\gamma$ vector could make the elimination of the latent HSV-1 in the TG possible.

In this study, we cloned the human IFN $\gamma$ gene and incorporated it into the rdHSV-IFN $\gamma$ vector. Previous studies ${ }^{28,29}$ have reported that IFN $\gamma$ always has both antiviral and immunoregulatory roles in virus-infected cells and the intercellular substance. Upstream of the therapeutic gene insertion location in the rdHSV-IFN $\gamma$ vector, we added a strong promoter, $\mathrm{CMV}$, to ensure sufficient expression of the therapeutic gene. Our results also confirmed that IFN $\gamma$ was expressed in the TG where it could carry out its antiviral therapy and immunoregulatory functions.

\section{Conclusion}

Overall, the results confirm that rdHSV-IFN $\gamma$ can act as a vector to carry therapeutic genes to regions with the latent HSV-1 infection and to trigger the expression of the therapeutic genes (eg, the TG in the HSK model). The rdHSV-IFN $\gamma$ vector carrying therapeutic genes may become a promising method to inhibit the reactivation of the latent HSV-1 and to prevent the recurrence of the HSK in clinical practice; however, much more evidence is required before the clinical application of rdHSV-IFN $\gamma$ becomes possible. We are planning to investigate the antiviral effects of rdHSV-IFN $\gamma$ against various HSV-1 strains in this rat model system.

\section{Acknowledgment}

This research was supported by the Science and Technology Research and Development Program of Shaanxi Province, People's Republic of China (Grant No 2011K12-55).

\section{Disclosure}

The authors report no conflicts of interest in this work.

\section{References}

1. Lin T, Gong L, Sun XH, et al. Effectiveness and safety of $0.5 \%$ ganciclovir in situ ophthalmic gel for herpes simplex keratitis, a multicenter, randomized, investigator-masked, parallel group study in Chinese patients. Drug Des Devel Ther. 2013;7:361-368.

2. Chen D, Zhang D, Xu L, et al. The alterations of matrix metalloproteinase-9 in mouse brainstem during herpes simplex virus type 1-induced facial palsy. J Mol Neurosci. 2013;51(3):703-709.

3. Alekseev O, Donovan K, Zaizkhan-Clifford J. Inhibition of ataxia telangiectasia mutated (ATM) kinase suppresses herpes simplex virus type 1 (HSV-1) keratitis. Invest Ophthalmol Vis Sci. 2014;55(2):706-715.

4. Messer HG, Jacobs D, Dhummakupt A, et al. Inhibition of H3K27me3specific histone demethylases JMJD3 and UTX blocks reactivation of herpes simplex virus 1 in trigeminal ganglion neurons. $J$ Virol. 2015; 89(6):3417-3420.

5. Sheppard JD, Wertheimer ML, Scoper SV. Modalities to decrease stromal herpes simplex keratitis reactivation rates. Arch Ophthalmol. 2009;127(7):852-856.

6. Arefian E, Bamdad T, Soleimanjahi H, et al. A kinetic study of gamma interferon production in herpes simplex virus-1 DNA prime-protein boost regimen comparing to DNA or subunit vaccination. Mol Biol (Mosk). 2009;43(3):388-393.

7. Hu K, Dou J, Yu F, et al. An ocular mucosal administration of nanoparticles containing DNA vaccine Prsc-Gd-IL-21 confers protection against mucosal challenge with herpes simplex virus type 1 in mice. Vaccine. 2011;29(7):1455-1462.

8. Theopold CP, Gheerardyn R, Bleiziffer O, et al. A novel replicationdefective HSV-1 vector for regulatable gene delivery to wounds. $J \mathrm{Am}$ Coll Surg. 2004;199(3):57-58.

9. Palmer JA, Branston RH, Lilley CE, et al. Development and optimization of herpes simplex virus vectors for multiple long-term gene delivery to the peripheral nervous system. $J$ Virol. 2000;74:5604-5618.

10. Anesti AM, Peeters PJ, Royaux I, et al. Efficient delivery of RNA interference to peripheral neurons in vivo using herpes simplex virus. Nucleic Acids Res. 2008;36(14):e86.

11. Xu K, Liu XN, Zhang HB, et al. Replication-defective HSV-1 effectively targets targets trigeminal ganglion and inhibits viral pathopoiesis by mediating interferon gamma expression in SH-SY5Y cells. $J \mathrm{Mol}$ Neurosci. 2014;53(1):78-86.

12. Xu K, Wang X, Shi Q, et al. Human prion protein mutants with deleted and inserted octarepeats undergo different pathways to trigger cell apoptosis. J Mol Neurosci. 2011;43(3):225-234.

13. Chiang LC, Chiang W, Chang MY, et al. Antiviral activity of Plantago major extracts and related compounds in vitro. Antiviral Res. 2002; 55(1):53-62.

14. Wang X, Shi Q, Xu K, et al. Familial CJD associated PrP mutants within transmembrane region induced Ctm-PrP retention in ER and triggered apoptosis by ER stress in SH-SY5Y cells. PLoS One. 2011;6(1):e14602.

15. Shahidi Bonjar MR, Shahidi Bonjar L. Design of a new therapy for patients with chronic kidney disease: use of microarrays for selective hemoadsorption of uremic wastes and toxins to improve homeostasis. Drug Des Devel Ther. 2015;9:625-629.

16. Whitehead JL, Ohara PT, Tauscher AN, et al. A procedure to deliver simplex virus to the murine trigeminal ganglion. Brain Res Brain Res Protoc. 2003;12(1):60-66.

17. Meunier A, Latremoliere A, Mauborgne A, et al. Attenuation of pain-related behavior in a rat model of trigeminal neuropathic pain by viral-driven enkephalin overproduction in trigeminal ganglion neurons. Mol Ther. 2015;11(4):608-616.

18. Goins WF, Cohen JB, Glorioso JC. Gene therapy for the treatment of chronic peripheral nervous system pain. Neurobiol Dis. 2012;48(2): 255-270. 
19. Farooq AV, Shukla D. Herpes simplex epithelial and stromal keratitis: an epidemiologic update. Surv Ophthalmol. 2012;57(5):448-462.

20. vanVelzen M, van Loenen FB, Meesters RJ, et al. Latent acyclovirresistant herpes simplex virus type 1 in trigeminal ganglia of immunocompetent individuals. J Infect Dis. 2012;205(10):1539-1543.

21. Bieber T, Chosidow O, Bodsworth N, et al. Efficacy and safety of acyclovir mucoadhesive buccal tablet in immunocompetent patients with labial herpes: a double-blind placebo-controlled, self-initiated trial. J Drugs Dermatol. 2014;13(7):791-798.

22. Colin J, Prisant O, Cochener B, et al. Comparison of the efficacy and safety of valaciclovir and acyclovir for the treatment of herpes zoster ophthalmicus. Ophthalmology. 2000;107(8):1507-1511.

23. Turner LD, Beckingsale P. Acyclovir-resistant herpetic keratitis in a solid-organ transplant recipient on systemic immunosuppression. Clin Ophthalmol. 2013;7:229-232.

24. Sia KC, Wang GY, Ho IA, et al. Optimal purification method for herpesbased viral vectors that confers minimal cytotoxicity for systemic route of vector administration. $J$ Virol Methods. 2007;139(2):166-174.
25. Moriuchi S, Krisky DM, Marconi PC, et al. HSV vector cytotoxicity is inversely correlated with effective TK/GCV suicide gene therapy of rat gliosarcoma. Gene Ther. 2000;7(17):1483-1490.

26. Goel N, Rong Q, Zimmerman D, et al. ALEAPS heteroconjugate vaccine containing a T cell epitope from HSV-1 glycoprotein D elicits Th1 responses and protection. Vaccine. 2003;21(27-30):4410-4420.

27. Quenelle DC, Collins DJ, Rice TL, et al. Effect of an immune enhancer, GPI-100, on vaccination with live attenuated herpes simplex virus type 2 or glycoprotein D on genital HSV-2 infections of guinea pigs. Antiviral Res. 2008;80(2):223-224.

28. Fekete T, Pazmandi K, Szabo A, et al. The antiviral immune response in human conventional dendritic cells is controlled by the mammalian target of rapamycin. J Leukoc Biol. 2014;96(4):579-589.

29. Wang LC, Cheng SO, Chang SP, et al. Enterovirus 71 proteins $2 \mathrm{~A}$ and 3D antagonize the antiviral activity of gamma interferon via signaling attenuation. J Virol. 2015;89(14):7028-7037.
Drug Design, Development and Therapy

\section{Publish your work in this journal}

Drug Design, Development and Therapy is an international, peerreviewed open-access journal that spans the spectrum of drug design and development through to clinical applications. Clinical outcomes, patient safety, and programs for the development and effective, safe, and sustained use of medicines are a feature of the journal, which

\section{Dovepress}

has also been accepted for indexing on PubMed Central. The manuscript management system is completely online and includes a very quick and fair peer-review system, which is all easy to use. Visit http://www.dovepress.com/testimonials.php to read real quotes from published authors.

Submit your manuscript here: http://www.dovepress.com/drug-design-development-and-therapy-journal 